PROCEEDINGS OF THE

AMERICAN MATHEMATICAL SOCIETY

Volume 135, Number 7, July 2007, Pages 2055-2058

S 0002-9939(07)08700-X

Article electronically published on January 9, 2007

\title{
PERIOD TWO IMPLIES CHAOS FOR A CLASS OF ODES
}

\author{
FRANCO OBERSNEL AND PIERPAOLO OMARI
}

(Communicated by Carmen C. Chicone)

\begin{abstract}
We extend a result of J. Andres and K. Pastor concerning scalar time-periodic first order ordinary differential equations without uniqueness, by proving that the existence of just one subharmonic implies the existence of large sets of subharmonics of all given orders. Since these periodic solutions must coexist with complicated dynamics, we might paraphrase T. Y. Li and J. A. Yorke by loosely saying that in this setting even period two implies chaos. Similar results are obtained for a class of differential inclusions.
\end{abstract}

Let us consider the first order scalar ordinary differential equation

$$
x^{\prime}=f(t, x),
$$

where $f: \mathbb{R} \times \mathbb{R} \rightarrow \mathbb{R}$ is periodic in $t$ with period 1 and satisfies the $L^{1}$-Carathéodory conditions. We recall that, given $n \in \mathbb{N}$, with $n>1$, a subharmonic solution of (11) of order $n$ is a periodic solution of (1) of minimum period $n$. Since in this setting uniqueness for the Cauchy problem is not guaranteed, existence of subharmonic solutions may occur (a simple explicit example is given in [6, Example 3.3]).

In the recent paper [3, J. Andres and K. Pastor extend a previous result in 2 concerning a version of the Sharkovskii cycle coexistence theorem for multivalued $M$-maps. In particular, they prove that, if a $M$-map has a $n$-orbit, then it also has a $k$-orbit, for every $k \triangleleft n$, with at most two exceptions (here $\triangleleft$ denotes the Sharkovskii order [8]). The occurrence of exceptions in the context of $M$-maps is witnessed by some examples in [1. As an application, described in [3. Section 3], they obtain the following result concerning the ordinary differential equation (11).

Theorem 1 ([3, Theorem 4]). Assume that $f$ is continuous and all solutions of equation (11) extend to $\mathbb{R}$. If (1) has an n-periodic solution, then it also admits $k$-periodic solutions, for every $k \triangleleft n$, with at most two exceptions. In particular, if $n \neq 2^{m}$ for all $m \in \mathbb{N}$, then infinitely many (subharmonic) periodic solutions of (1) coexist.

We will show that the existence of just one subharmonic of order $n>1$ is sufficient to imply the existence of large sets of subharmonic solutions of all orders

Received by the editors February 1, 2006 and, in revised form, February 28, 2006.

2000 Mathematics Subject Classification. Primary 34C25, 34A60.

Key words and phrases. First order scalar ordinary differential equation, periodic solution, subharmonic solution, lower and upper solutions, differential inclusion.

The first author acknowledges the support of G.N.A.M.P.A., in the setting of the project "Soluzioni periodiche di equazioni differenziali ordinarie".

The second author acknowledges the support of M.I.U.R, in the setting of the P.R.I.N. project "Equazioni differenziali ordinarie e applicazioni".

(C)2007 American Mathematical Society Reverts to public domain 28 years from publication 
$k \in \mathbb{N}^{+}$, with no exceptions and no relation to Sharkovskii order. We also demonstrate that, in this case, the situation is much more complicated; indeed, every possible discrete dynamics can be realized by a solution of (1), in a sense that will be formalized.

Theorem 2. If there is a subharmonic solution of (11) of order $n>1$, then, for every $k>1$, there exists a subharmonic solution of (11) of order $k$. In addition, the set $\mathcal{X}_{k}$ of all subharmonic solutions of (1) of order $k$ has dimension at least $k$ as a subset of $L^{\infty}(\mathbb{R})$.

Proof. Let $x$ be a subharmonic solution of (11) of order $n>1$. Let $t_{0} \in \mathbb{R}$ be such that $x\left(t_{0}\right)<x\left(t_{0}+1\right)$. Let $j \in\{1, \ldots, n-1\}$ be such that

$$
x\left(t_{0}\right)<x\left(t_{0}+1\right) \leq \cdots \leq x\left(t_{0}+j\right) \text { and } x\left(t_{0}+j\right)>x\left(t_{0}+j+1\right) .
$$

Also, let $\ell \in\{0, \ldots, j-1\}$ be such that

$$
x\left(t_{0}+\ell\right)<x\left(t_{0}+\ell+1\right)=x\left(t_{0}+j\right) .
$$

Then we have $\max \left\{x\left(t_{0}+\ell\right), x\left(t_{0}+j+1\right)\right\}<x\left(t_{0}+j\right)$. Set

$$
I=] \max \left\{x\left(t_{0}+\ell\right), x\left(t_{0}+j+1\right)\right\}, x\left(t_{0}+j\right)[.
$$

Choose $p \in I$ and define $\alpha_{p}:\left[t_{0}+\ell, t_{0}+\ell+1\left[\rightarrow \mathbb{R}, \beta_{p}:\left[t_{0}+j, t_{0}+j+1[\rightarrow \mathbb{R}\right.\right.\right.$ by setting

$$
\begin{array}{lll}
\alpha_{p}\left(t_{0}+\ell\right)=p, & \alpha_{p}(t)=x(t) & \text { on }] t_{0}+\ell, t_{0}+\ell+1[, \\
\beta_{p}\left(t_{0}+j\right)=p, & \beta_{p}(t)=x(t) & \text { on }] t_{0}+j, t_{0}+j+1[.
\end{array}
$$

Then the 1-periodic extensions of $\alpha_{p}$ and $\beta_{p}$ onto $\mathbb{R}$ are, respectively, a lower solution and an upper solution of the 1-periodic problem associated with (1), according to the definition given, e.g., in [6, Section 2]. Hence, by [6, Theorem 2.1], for any such $p$, there exists a 1-periodic solution $u_{p}$ of (11) such that

$$
\min \left\{\alpha_{p}, \beta_{p}\right\} \leq u_{p} \leq \max \left\{\alpha_{p}, \beta_{p}\right\} .
$$

Note that there exists $s \in] t_{0}, t_{0}+1\left[\right.$ such that $\alpha_{p}(s)=\beta_{p}(s)$ and therefore $u_{p}(s)=$ $u_{q}(s)$ for all $p, q \in I$. By the lattice structure of the set of solutions of (1), we can easily find an increasing sequence $\left(u_{m}\right)_{m}$ of 1-periodic solutions of (11) such that $u_{m}\left(t_{0}\right)<u_{m+1}\left(t_{0}\right)$ and $u_{m}(s)=u_{0}(s)$ for every $m$.

A subharmonic solution $v$ of (11) of order $k$ can be constructed as follows: we define a $k$-periodic solution $v$ of (1) by setting $v(t)=u_{m}(t)$ on $[s+m+i k, s+m+$ $1+i k[$ for every $m \in\{0, \ldots, k-1\}$ and $i \in \mathbb{Z}$. Let us prove that $v$ has minimum period $k$. Since $v$ is continuous and non-constant, $v$ has a minimum period $T>0$. Suppose, by contradiction, that $T<k$. Note that, by definition of $v, T \neq 1$ and, as $k$ is a multiple of $T, T \leq k / 2$. This implies in particular that $T<k-1$. If $T>1$ (and hence $k>2$ ), we get

$$
\max v=\left.\max v\right|_{[s, s+T]} \leq \max _{m=0, \ldots, k-2} \max u_{m}=\max u_{k-2}<\max u_{k-1}=\max v .
$$

Whereas if $T<1$, we get

$$
\max v=\left.\max u_{0}\right|_{[s, s+T]} \leq \max u_{0}<\max u_{k-1}=\max v .
$$

In both cases a contradiction is achieved. Hence we conclude that $T=k$.

Finally, to prove that the dimension of $\mathcal{X}_{k}$ is at least $k$, we show that $[0,1]^{k}$ is embedded into $\mathcal{X}_{k}$. By [6. Theorem 3.5] the set $\mathcal{K}_{m}$ of all 1-periodic solutions $v$ of (11), with $u_{m} \leq v \leq u_{m+1}$, is a non-degenerate continuum in $L^{\infty}(\mathbb{R})$. Let $\mathcal{T}_{m}$ be a 
totally ordered subset of $\mathcal{K}_{m}$. By [6, Lemma 3.6] $\mathcal{T}_{m}$ is homeomorphic to a compact interval of $\mathbb{R}$. Define

$$
\Phi_{k}: \prod_{m=0}^{k-1} \mathcal{T}_{m} \rightarrow L^{\infty}(\mathbb{R})
$$

by setting

$$
\Phi_{k}\left(v_{0}, \ldots, v_{k-1}\right)(t)=v_{m}(t) \quad \text { on }[s+m+i k, s+m+1+i k[
$$

for every $m \in\{0, \ldots, k-1\}$ and $i \in \mathbb{Z}$. Clearly $\Phi_{k}$ is one-to-one and continuous, and hence it is a homeomorphism between $\prod_{m=0}^{k-1} \mathcal{T}_{m}$ and $\Phi\left(\prod_{m=0}^{k-1} \mathcal{T}_{m}\right) \subseteq \mathcal{X}_{k}$.

Remark 1. We note that our approach, which is based on the use of (non-ordered) lower and upper solutions, does not require the hypothesis in Theorem 1 that all solutions of (1) exist on all of $\mathbb{R}$.

Remark 2. We see from Theorem 2 that the existence of at least one subharmonic solution of (11) of order $n>1$ implies the existence of an infinite-dimensional set of subharmonics (of any given order). Yet, this is only one indication of the complexity of the dynamics in this situation. In the proof of Theorem 2 we proved that, for each $p \in I$, there exists a 1-periodic solution $u_{p}$ of (11) such that $u_{p}\left(t_{0}\right)=p$. Furthermore all these solutions attain the same value at $s$. Fix an arbitrary function $\varphi: I \rightarrow I$. Then, for each $p \in I$, there exists a solution $u_{\varphi, p}$ of (1) on $\mathbb{R}$ such that $u_{\varphi, p}\left(t_{0}\right)=p$ and $u_{\varphi, p}\left(t_{0}+k\right)=\varphi^{k}(p)$ for all $k \in \mathbb{N}$. This is an easy consequence of $\underline{6}$, Theorem 2.1]. For this reason, we may say that the solutions $\left\{u_{\varphi, p} \mid p \in I\right\}$ "realize" the dynamics of $\varphi$. Accordingly, all possible discrete dynamics can be realized by a solution of (11). In particular, as explained in [6, Theorem 3.6], we find an infinitedimensional set $\mathcal{X} \subset L^{\infty}(\mathbb{R})$ such that each $z \in \mathcal{X}$ is a (Bohr) almost periodic solution of (1), which is not periodic.

Remark 3. The authors of [3] extend the conclusions of Theorem 1 to a class of differential inclusions such as

$$
x^{\prime} \in F(t, x) .
$$

This is done in [3, Theorem 5]. They assume that $F: \mathbb{R} \times \mathbb{R} \rightarrow 2^{\mathbb{R}}$ is a set-valued map having non-empty compact convex values, which is periodic in $t$ with period 1 and satisfies the following conditions:

(i) $F(\cdot, x)$ is measurable for every $x \in \mathbb{R}$,

(ii) $F(t, \cdot)$ is upper semicontinuous for a.e. $t \in[0,1]$,

(iii) there exist $a, b \in \mathbb{R}$ such that $|F(t, x)| \leq a+b|x|$ for a.e. $t \in[0,1]$ and every $x \in \mathbb{R}$.

We point out that we are also able to extend the validity of our Theorem 2 to the differential inclusion (2), under the above assumptions on $F$, but with condition (iii) replaced by the weaker assumption

(iii') for each $\rho>0$ there exists $\gamma \in L^{1}(0,1)$ such that $|F(t, x)| \leq \gamma(t)$ for a.e. $t \in[0,1]$ and every $x \in[-\rho, \rho]$.

We omit the proof of this result since it follows the argument of the proof of Theorem 2 with just a few minor changes; in particular, the use of Theorem 2.1 in [6] is replaced here by a slightly more lengthy direct construction relying on Theorem 6 in [4, Chapter 2.7]. (The interested reader can find a detailed proof in [7.) 
Accordingly, even in the setting of (2), we can conclude that the existence of one subharmonic of order $n \geq 2$ implies the existence of subharmonics of all orders.

\section{REFERENCES}

[1] J. Andres, J. Fišer and L. Jüttner, On a multivalued version of the Sharkovskii Theorem and its application to differential inclusions, Set-Valued Anal. 10 (2002), 1-14. MR1888453 (2002m:37057)

[2] J. Andres, L. Jüttner and K. Pastor, On a multivalued version of the Sharkovskii Theorem and its application to differential inclusions II, Set-Valued Anal. 13 (2005), 47-68. MR 2128697 (2006c:37018)

[3] J. Andres and K. Pastor, A version of Sharkovskii Theorem for differential equations, Proc. Amer. Math. Soc. 133 (2005), 449-453. MR2093067 (2005e:34124)

[4] A. F. Filippov, Differential Equations with Discontinuous Righthand Sides, Kluwer Academic Publishers, Dordrecht, 1988. MR 1028776 (90i:34002)

[5] T.Y. Li and J.A. Yorke, Period three implies chaos, Amer. Math. Monthly 82 (1975), 985992. MR0385028 (52:5898)

[6] F. Obersnel and P. Omari, Old and new results for first order periodic ODEs without uniqueness: a comprehensive study by lower and upper solutions, Advanced Nonlinear Studies 4 (2004), 323-376. MR2079818 (2005g:34093)

[7] F. Obersnel and P. Omari, Period two implies any period for a class of differential inclusions, Quaderni Matematici Ser. II Univ. Trieste 575, 2006. (http://www.dmi.units.it/ pubblicazioni/Quaderni_Matematici/2006.html)

[8] A. N. Sharkovskii, Coexistence of cycles of a continuous map of a line into itself (Russian), Ukrainian Math. J. 16 (1964), 61-71. MR.0159905 (28:3121)

Dipartimento di Matematica e Informatica, Università degli Studi di Trieste, Via A. VAlerio 12/1, I-34127 Trieste, Italy

E-mail address: obersnel@units.it

Dipartimento di Matematica e Informatica, Università degli Studi di Trieste, Via A. VAlerio 12/1, I-34127 Trieste, Italy

E-mail address: omari@units.it 